\title{
Directed Ortho-Metalation of Unprotected Benzoic Acids. Methodology and Regioselective Synthesis of Useful Contiguously 3- and 6-Substituted 2- Methoxybenzoic acid Building Blocks
}

\author{
SUPPORTING INFORMATION
}

Thi-Huu Nguyen, Anne-Sophie Castanet, and Jacques Mortier*

\begin{abstract}
Université du Maine and CNRS, Unité de Chimie Organique Moléculaire et Macromoléculaire (UMR 6011), Faculté des Sciences, avenue Olivier Messiaen, 72085 Le Mans Cedex 9 (France)
\end{abstract}




\section{Table of contents}

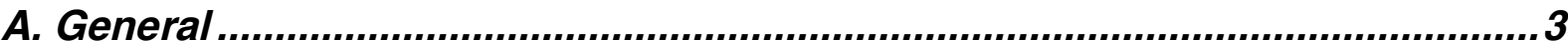

B. General procedure for the preparation of 6-substituted 2-methoxybenzoic acids (9a-f,i,j). Preparation of 3-hydroxy-7-methoxyisobenzofuran-1(3H)-one (16) and 7-methoxy-3-phenylisobenzofuran-1(3H)-one (17) ............................... 3

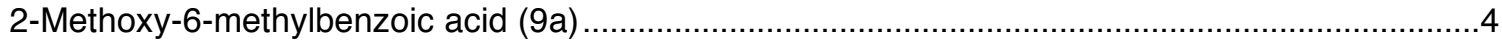

2-Methoxy-6-(trimethylsilyl)benzoic acid (9b) (in situ quench technique) .......................................

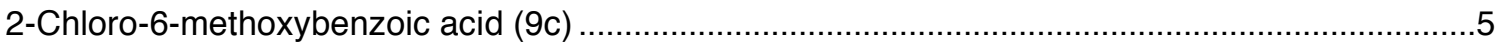

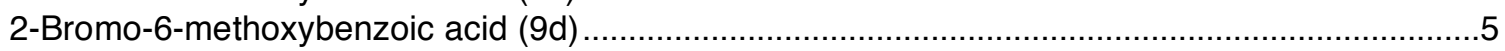

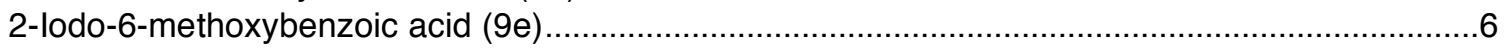

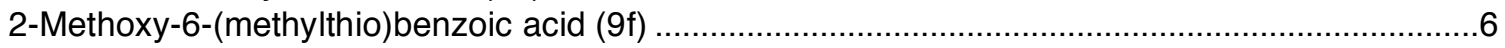

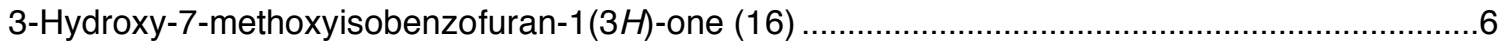

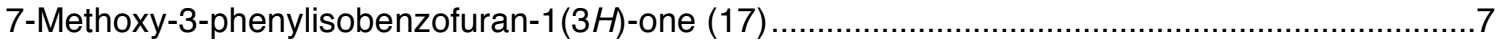

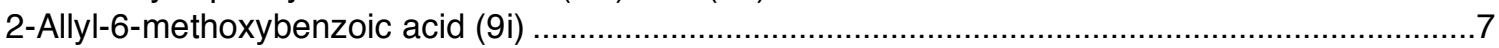

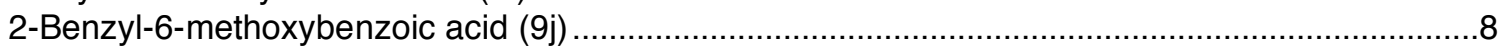

C. General procedure for the preparation of 3-substituted 2-methoxybenzoic

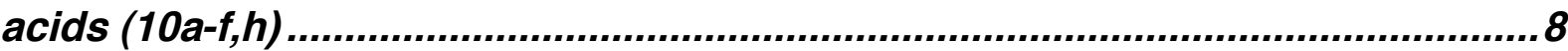

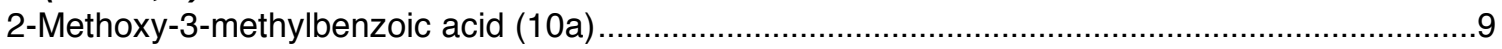

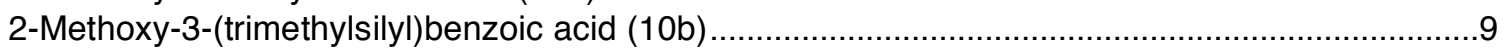

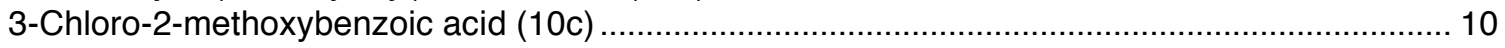

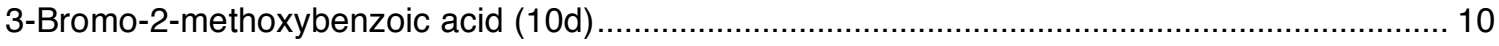

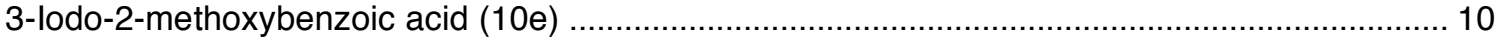

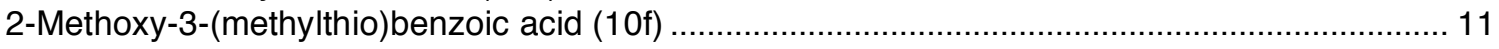

3-(Hydroxy(phenyl)methyl)-2-methoxybenzoic acid (10h) ....................................................... 11

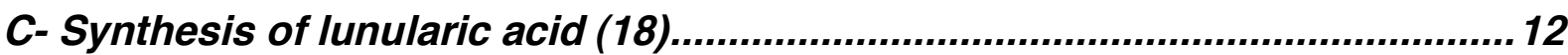

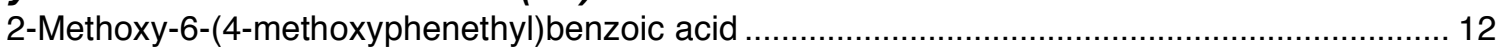

2-Hydroxy-6-(4-hydroxyphenethyl)benzoic acid (lunularic acid (18)) .......................................... 12 


\section{A. General}

For standard working practice, see refs ${ }^{(1,2)}$. Reactions were carried out under argon in oven-dried glassware. Tetrahydrofuran was dried from sodium benzophenone. lodomethane, $\mathrm{N}, \mathrm{N}$-dimethylformamide and benzaldehyde were dried with $\mathrm{CaH}_{2}$ and distilled prior to use. NMR spectra were recorded on a 200- or 400$\mathrm{MHz}$ spectrometer. ${ }^{13} \mathrm{C}$ NMR spectra were obtained with broadband proton decoupling. For spectra recorded in $\mathrm{CDCl}_{3}$, chemical shifts were recorded relative to the internal TMS (tetramethylsilane) reference signal. For acetone- $d_{6}$, chemical shifts are given relative to the solvent signals. All melting points are uncorrected.

$n$-BuLi (1.6 M in hexanes) and s-BuLi (1.3 $\mathrm{M}$ in cyclohexane-hexanes) were titrated periodically against 2,5-dimethoxybenzyl alcohol. $N, N, N^{\prime}, N^{\prime}$-Tetramethyl-1,2ethylenediamine (TMEDA) was distilled from $\mathrm{CaH}_{2}$. Potassium tert-butylate $(t$-BuOK) was sublimated.

\section{B. General procedure for the preparation of 6-substituted 2-methoxybenzoic acids (9a-f,i,j). Preparation of 3-hydroxy-7-methoxyisobenzofuran-1(3H)-one} (16) and 7-methoxy-3-phenylisobenzofuran-1(3H)-one (17)

To a stirred solution of a 1:1 s-BuLi/TMEDA complex $(11 \mathrm{mmol})$ in anhydrous THF at $-78^{\circ} \mathrm{C}$ was added dropwise under argon the recrystallized 2-methoxybenzoic acid (11) $(0.76 \mathrm{~g}, 5 \mathrm{mmol})$ dissolved in dry THF $(5 \mathrm{~mL})$. After $2 \mathrm{~h}$ at this temperature, the mixture was treated with an excess of the appropriate electrophile $(20 \mathrm{mmol})$ dissolved in THF. The resulting solution was then slowly allowed to warm to ambient temperature, and water was added. The aqueous layer was washed with diethyl ether $(2 \times 30 \mathrm{~mL})$, and shaken, and acidified with $2 \mathrm{M} \mathrm{HCl}$. The mixture was then diluted with diethyl ether and the organic layer was separated and dried with $\mathrm{MgSO}_{4}$.

Schlosser, M. Organometallics in Synthesis. A Manual; Wiley: Chichester, $2^{\text {nd }}$ Edition, 2002.

(a) Cantegril, R.; Mortier, J.; Croisat, D.; Peignier, R. Wo. Pat. 9602138, 1996. (b) Cantegril, R.; Croisat, D.; Desbordes, P.; Guigues, F.; Mortier, J.; Peignier, R.; Vors, J.-P. U. S. Pat. 5945382, 1999. 
Filtration and concentration in vacuo gave the crude benzoic acids, which were purified by recrystallization or chromatography for characterisation in each case.

\section{2-Methoxy-6-methylbenzoic acid (9a)}

According to the general procedure, the recrystallized 2-methoxybenzoic acid $(11,0.76 \mathrm{~g}, 5 \mathrm{mmol})$ in THF was added dropwise to a stirred solution of $s$ BuLi/TMEDA (11 mmol). The solution was quenched by Mel (2.84 g, $20 \mathrm{mmol})$. Workup in the usual manner followed by recrystallization (heptane/ethylacetate) afforded $9 \mathrm{a}(0.51 \mathrm{~g}, 61 \%)$ as a white solid: $\mathrm{mp} 138.5-139.0^{\circ} \mathrm{C}\left(\right.$ lit. $\left.^{(3)} 137-138^{\circ} \mathrm{C}\right)$. ${ }^{1} \mathrm{H}$ NMR (200 MHz, $\left.\mathrm{CDCl}_{3}\right) \delta \mathrm{ppm:} 2.45(\mathrm{~s}, 3 \mathrm{H}), 3.88(\mathrm{~s}, 3 \mathrm{H}), 6.82(\mathrm{~d}, 1 \mathrm{H}, J=8.0 \mathrm{~Hz})$, $6.83(\mathrm{~d}, 1 \mathrm{H}, J=8.0 \mathrm{~Hz}), 7.29(\mathrm{t}, 1 \mathrm{H}, J=J=8.0 \mathrm{~Hz}) \cdot{ }^{13} \mathrm{C} \mathrm{NMR}\left(100 \mathrm{MHz}, \mathrm{CDCl}_{3}\right) \delta$ ppm: 20.6, 56.6, 109.2, 122.1, 123.6, 131.6, 138.7, 157.4, 173.1. IR (neat): 2995, $1692,1585,1471,1267,1087,915 \mathrm{~cm}^{-1}$. Anal. Calcd for $\mathrm{C}_{9} \mathrm{H}_{10} \mathrm{O}_{3}: \mathrm{C}, 65.05 ; \mathrm{H}, 6.07$. Found: C, 65.03; H, 6.05.

\section{2-Methoxy-6-(trimethylsilyl)benzoic acid (9b) (in situ quench technique)}

To a solution of LTMP $(9 \mathrm{mmol})$ in THF $(20 \mathrm{~mL})$ at $-78{ }^{\circ} \mathrm{C}$ were added successively chlorotrimethylsilane $(1.38 \mathrm{~mL}, 10.5 \mathrm{mmol})$ in THF $(5 \mathrm{~mL})$ and 2methoxybenzoic acid (6) (0.46 g, $3 \mathrm{mmol})$ in THF (3 mL). After being warmed gradually to rt for $4 \mathrm{~h}$, the solution was allowed to warm to rt. Aqueous $2 \mathrm{M} \mathrm{NaOH}$ was added until the $\mathrm{pH}$ reached 10 . The aqueous layer was washed with diethyl ether, acidified with aqueous $4 \mathrm{M} \mathrm{HCl}$, and extracted with diethyl ether. The organic layer was dried over $\mathrm{MgSO}_{4}$, filtrated, and concentrated in vacuo. The residue was purified by colum chromatography on silicagel (cyclohexane/diethyl ether 90:10) to give $\mathbf{9 b}$ $(0.60 \mathrm{~g}, 89 \%)$ as a white solid (mp 85.5-87.0 $\left.{ }^{\circ} \mathrm{C}\right) .{ }^{1} \mathrm{H}$ NMR $\left(200 \mathrm{MHz}, \mathrm{CDCl}_{3}\right) \delta \mathrm{ppm}$ : 0.33 (s, 9H), 3.98 (s, 3H), 7.05 (d, 1H, J=8.4 Hz), 7.37 (d, 1H, J= $7.8 \mathrm{~Hz}$ ), 7.51 (dd, $1 \mathrm{H}, J=8.4 \mathrm{~Hz}, J=7.8 \mathrm{~Hz}) \cdot{ }^{13} \mathrm{C} \mathrm{NMR}\left(100 \mathrm{MHz}, \mathrm{CDCl}_{3}\right) \delta \mathrm{ppm}: 0.6(3 \mathrm{C}), 56.0,112.1$, 124.2, 127.9, 131.8, 144.4, 157.2, 170.3. IR (neat): 2840, 1681, 1571, 1445, 1243,

3 Reitz, D. B.; Massey, S. M. J. Org. Chem. 1990, 55, 1375. 
1126, $950 \mathrm{~cm}^{-1}$. Anal. calcd. for $\mathrm{C}_{11} \mathrm{H}_{16} \mathrm{O}_{3} \mathrm{Si}$ : C, 58.89; $\mathrm{H}, 7.19$. Found: $\mathrm{C}, 58.94 ; \mathrm{H}$, 7.12 .

\section{2-Chloro-6-methoxybenzoic acid (9c)}

According to the general procedure, the recrystallized 2-methoxybenzoic acid $(11,0.76 \mathrm{~g}, 5 \mathrm{mmol})$ in THF was added dropwise to a stirred solution of $s$ BuLi/TMEDA (11 mmol). The solution was quenched by hexachloroethane (4.73 g, $20 \mathrm{mmol})$. Workup in the usual manner followed by chromatography (cyclohexane/ethylacetate 80:20) afforded 9c (0.54 g, 58\%) as a white solid: $\mathrm{mp}$ 142.5-144 ${ }^{\circ} \mathrm{C}$ ( Lit. $\left.^{(4)} 140-141^{\circ} \mathrm{C}\right) .{ }^{1} \mathrm{H}$ NMR (200 MHz, $\left.\mathrm{CDCl}_{3}\right) \delta$ ppm: 3.88 (s, 3H), $6.88(\mathrm{dd}, 1 \mathrm{H}, J=8.4 \mathrm{~Hz}, J=0.8 \mathrm{~Hz}), 7.03$ (dd, $1 \mathrm{H}, J=8.2 \mathrm{~Hz}, J=0.8 \mathrm{~Hz}$ ), 7.29 (dd, $1 \mathrm{H}, J=8.4 \mathrm{~Hz}, J=8.2 \mathrm{~Hz}) \cdot{ }^{13} \mathrm{C}$ NMR $\left(200 \mathrm{MHz}, \mathrm{CDCl}_{3}\right) \delta \mathrm{ppm:} \mathrm{56.1,} \mathrm{110.4,} \mathrm{120.9,}$ 124.9, 129.2, 130.9, 156.3, 165.9. IR (neat): 2839, 2531, 1713, 1589, 1467, 1262, 1029, $924 \mathrm{~cm}^{-1}$. Anal. Calcd for $\mathrm{C}_{8} \mathrm{H}_{7} \mathrm{ClO}_{3}: \mathrm{C}, 51.50 ; \mathrm{H}, 3.78$. Found: $\mathrm{C}, 51.81 ; \mathrm{H}$, 3.71 .

\section{2-Bromo-6-methoxybenzoic acid (9d)}

According to the general procedure, the recrystallized 2-methoxybenzoic acid $(11,0.76 \mathrm{~g}, 5 \mathrm{mmol})$ in THF was added dropwise to a stirred solution of $s$ BuLi/TMEDA (11 mmol). The solution was quenched by dibromotetrachloroethane $(6.51 \mathrm{~g}, 20 \mathrm{mmol})$. Workup in the usual manner followed by chromatography (cyclohexane/ethylacetate 80:20) afforded 9d (0.68 g, 59\%) as a white solid: mp 127$129^{\circ} \mathrm{C}$ (Lit. $\left.{ }^{(5)} 124-127^{\circ} \mathrm{C}\right) .{ }^{1} \mathrm{H}$ NMR $\left(200 \mathrm{MHz}, \mathrm{CDCl}_{3}\right) \delta$ ppm: 3.89 (s, 3H), 6.87 (dd, $1 \mathrm{H}, J=8.4 \mathrm{~Hz}, J=0.8 \mathrm{~Hz}), 7.02(\mathrm{dd}, 1 \mathrm{H}, J=8.1 \mathrm{~Hz}, J=0.8 \mathrm{~Hz}), 7.32(\mathrm{dd}, 1 \mathrm{H}, J=$ $8.4 \mathrm{~Hz}, J=8.1 \mathrm{~Hz}) .{ }^{13} \mathrm{C} \mathrm{NMR}\left(50 \mathrm{MHz}, \mathrm{CDCl}_{3}\right) \delta \mathrm{ppm}: 56.3,109.6,121.7,122.7$, 131.5, 131.6, 157.3, 171.3. IR (neat): 2841, 1719, 1589, 1469, $1262 \mathrm{~cm}^{-1}$.

Postmus, C. Jr.; Kaye, I. A.; Craig, C. A.; Matthews, R. S. J. Org. Chem. 1964, 29, 2693.

5 Sugaya, T.; Mimura, Y.; Kato, N.; Ikuta, M.; Mimura, T.; Kasai, M.; Tomioka, S. Synthesis 1994, 73. 


\section{2-lodo-6-methoxybenzoic acid (9e)}

According to the general procedure, the recrystallized 2-methoxybenzoic acid $(11,0.76 \mathrm{~g}, 5 \mathrm{mmol})$ in THF was added dropwise to a stirred solution of $s$ BuLi/TMEDA (11 mmol). The solution was quenched by iodine $(5.07 \mathrm{~g}, 20 \mathrm{mmol})$. Workup in the usual manner followed by chromatography (cyclohexane/ethylacetate 80:20) afforded 9 e (0.64 g, 46\%) as a brown solid: mp 129-131 (Lit. $\left.{ }^{(6)} 128-130{ }^{\circ} \mathrm{C}\right)$. ${ }^{1} \mathrm{H}$ NMR (200 MHz, CDCl $)_{3} 3.84(\mathrm{~s}, 3 \mathrm{H}), 6.92(\mathrm{dd}, 1 \mathrm{H}, J=8.4 \mathrm{~Hz}, J=0.9 \mathrm{~Hz}), 7.10$ (dd, $1 \mathrm{H}, J=8.4 \mathrm{~Hz}, J=8.1 \mathrm{~Hz}), 7.44(\mathrm{dd}, 1 \mathrm{H}, J=8.1 \mathrm{~Hz}, J=0.9 \mathrm{~Hz}) .{ }^{13} \mathrm{C}$ NMR $(100$ $\left.\mathrm{MHz}, \mathrm{CDCl}_{3}\right) \delta$ ppm: 56.6, 92.2, 110.9, 129.2, 131.2, 131.9, 156.7, 172.6. IR (neat): $2841,1719,1589,1469,1262 \mathrm{~cm}^{-1}$.

\section{2-Methoxy-6-(methylthio)benzoic acid (9f)}

According to the general procedure, the recrystallized 2-methoxybenzoic acid $(11,0.76 \mathrm{~g}, 5 \mathrm{mmol})$ in THF was added dropwise to a stirred solution of $s$ BuLi/TMEDA (11 mmol). The solution was quenched by dimethyl disulfide $(1.88 \mathrm{~g}$, $20 \mathrm{mmol}$ ). Workup in the usual manner followed by recrystallization (cyclohexane/ethylacetate) afforded $9 f(0.47 \mathrm{~g}, 47 \%)$ as a white solid: $\mathrm{mp} 182-184{ }^{\circ} \mathrm{C}$ $\left(\mathrm{Lit}^{(7)} \cdot 184-185{ }^{\circ} \mathrm{C}\right) .{ }^{1} \mathrm{H}$ NMR $\left(200 \mathrm{MHz}, \mathrm{CDCl}_{3}\right) \delta \mathrm{ppm}: 2.45(\mathrm{~s}, 3 \mathrm{H}), 3.98(\mathrm{~s}, 3 \mathrm{H}), 6.80$ (d, $1 \mathrm{H}, J=8.2 \mathrm{~Hz}), 6.98(\mathrm{~d}, 1 \mathrm{H}, J=8.2 \mathrm{~Hz}), 7.40(\mathrm{t}, 1 \mathrm{H}, J=8.2 \mathrm{~Hz}) .{ }^{13} \mathrm{C}$ NMR $(100$ $\left.\mathrm{MHz} \mathrm{CDCl}_{3}\right) \delta \mathrm{ppm}: 17.0,56.6,107.6,107.9,119.4,131.9,142.6,157.9,169.1 . \mathrm{IR}$ (neat): 2941, 1688, 1578, 1461, 1261, 1040, $927 \mathrm{~cm}^{-1}$. Anal. Calcd for $\mathrm{C}_{9} \mathrm{H}_{10} \mathrm{O}_{3} \mathrm{~S}: \mathrm{C}$, 54.53; H, 5.08. Found: C, 54.41; H, 5.01.

\section{3-Hydroxy-7-methoxyisobenzofuran-1(3H)-one (16)}

According to the general procedure, the recrystallized 2-methoxybenzoic acid $(11,0.76 \mathrm{~g}, 5 \mathrm{mmol})$ in THF was added dropwise to a stirred solution of $s$ BuLi/TMEDA (11 mmol). The solution was quenched by DMF (1.46 g, $20 \mathrm{mmol})$

6 Lewis, A.; Stefanuti, I.; Swain, S. A.; Smith, S. A.; Taylor, R. J. K. Org. Biomol. Chem. 2003, 1, 104.

7 Cabiddu, S.; Melis, S.; Piras, P. P.; Secci, M. J. Organometallic Chem. 1977, 132 (3), 321-326. 
(reverse addition). Workup in the usual manner followed by chromatography (cyclohexane/ethylacetate 70:30) afforded $16(0.39 \mathrm{~g}, 43 \%)$ as a white solid: mp 155$157^{\circ} \mathrm{C}\left(\mathrm{Lit}^{(8)}{ }^{(155-156}{ }^{\circ} \mathrm{C}\right) .{ }^{1} \mathrm{H}$ NMR $\left(200 \mathrm{MHz}, \mathrm{CDCl}_{3}\right) \delta \mathrm{ppm}: 3.99$ (s, 3H), 6.53 (s, 1H), $7.01(\mathrm{~d}, 1 \mathrm{H}, J=8.4 \mathrm{~Hz}$ ), $7.18(\mathrm{~d}, 1 \mathrm{H} J=7.4 \mathrm{~Hz}$ ), $7.53(\mathrm{dd}, 1 \mathrm{H}, J=8.4 \mathrm{~Hz}, J=$ $7.4 \mathrm{~Hz}) .{ }^{13} \mathrm{C} \mathrm{NMR}\left(100 \mathrm{MHz}, \mathrm{CDCl}_{3}\right) \delta$ ppm: 55.6, 96.5, 112.3, 115.1, 121.2, 130.6, 133.0, 158.0, 167.3. IR (neat): 2987, 1754, 1601, 1486, 1296, 1197, $1027 \mathrm{~cm}^{-1}$.

\section{7-Methoxy-3-phenylisobenzofuran-1(3H)-one (17)}

According to the general procedure, the recrystallized 2-methoxybenzoic acid $(11,0.76 \mathrm{~g}, 5 \mathrm{mmol})$ in THF was added dropwise to a stirred solution of $s$ BuLi/TMEDA (11 mmol). The solution was quenched by benzaldehyde $(2.12 \mathrm{~g}$, $20 \mathrm{mmol}$ ) (reverse addition). Workup in the usual manner followed by recrystallization (cyclohexane/ethylacetate 70:30) afforded $17(0.624 \mathrm{~g}, 52 \%)$ as a white solid: $\mathrm{mp}$ 136-138 ${ }^{\circ} \mathrm{C}$ (Lit. $\left.{ }^{(9)} 139-141.5^{\circ} \mathrm{C}\right) .{ }^{1} \mathrm{H}$ NMR $\left(200 \mathrm{MHz}, \mathrm{CDCl}_{3}\right) \delta$ ppm: $4.02(\mathrm{~s}, 3 \mathrm{H})$, $6.31(\mathrm{~s}, 1 \mathrm{H}), 6.84(\mathrm{~d}, 1 \mathrm{H}, J=7.6 \mathrm{~Hz}), 6.94(\mathrm{~d}, 1 \mathrm{H}, J=8.4 \mathrm{~Hz}), 7.25-7.39(\mathrm{~m}, 5 \mathrm{H})$,

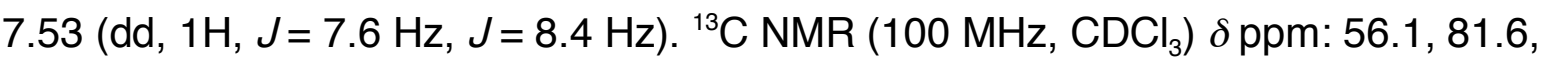
110.9, 112.9, 114.5, 126.9, 128.9 (2C), 129.1 (2C), 136.6, 136.7, 152.5, 158.5, 168.6. IR (neat): 2985, 1754, 1600, 1486, 1296, 1196, $1027 \mathrm{~cm}^{-1}$. Anal. Calcd for $\mathrm{C}_{15} \mathrm{H}_{12} \mathrm{O}_{3}$ : C, 74.99; H, 5.03. Found: C, 74.56; H, 5.03.

\section{2-Allyl-6-methoxybenzoic acid (9i)}

According to the general procedure, the recrystallized 2-methoxybenzoic acid $(11,0.76 \mathrm{~g}, 5 \mathrm{mmol})$ in THF was added dropwise to a stirred solution of $s$ BuLi/TMEDA (11 mmol). The solution was quenched by allyl bromide $(2.42 \mathrm{~g}$, $20 \mathrm{mmol}$ ) (reverse addition). Workup in the usual manner followed by chromatography (cyclohexane/ethylacetate $85: 15)$ afforded $9 \mathbf{i}(0.43 \mathrm{~g}, 45 \%)$ as a

Freskos, J. N.; Morrow, G. W. Swenton, J. S. J. Org. Chem. 1985, 50, 805-810.

Harland, P.; Hodge, P. Synthesis 1983, 419. 
white solid: $\mathrm{mp} 89-91^{\circ} \mathrm{C}\left(\right.$ Lit. $\left.^{(10)} 91^{\circ} \mathrm{C}\right) .{ }^{1} \mathrm{H}$ NMR $\left(200 \mathrm{MHz}, \mathrm{CDCl}_{3}\right) \delta$ ppm: 3.54 (d, $2 \mathrm{H}, J=6.7 \mathrm{~Hz}), 3.89(\mathrm{~s}, 3 \mathrm{H}), 5.04-5.15(\mathrm{~m}, 2 \mathrm{H}), 5.87-6.07(\mathrm{~m}, 1 \mathrm{H}), 6.85(\mathrm{~d}, 1 \mathrm{H}, J=$ $8.2 \mathrm{~Hz}), 6.91(\mathrm{~d}, 1 \mathrm{H}, J=7.8 \mathrm{~Hz}), 7.34(\mathrm{dd}, 1 \mathrm{H}, J=8.2 \mathrm{~Hz}, J=7.8 \mathrm{~Hz}) .{ }^{13} \mathrm{C} \mathrm{NMR}$ $\left(100 \mathrm{MHz}, \mathrm{CDCl}_{3}\right) \delta$ ppm: 37.9, 56.1, 109.3, 116.4, 122.1, 122.3, 131.2, 136.4, 139.6, 156.8, 172.9. IR (neat): 2951, 1685, 1581, 1471, 1289, 1061, $918 \mathrm{~cm}^{-1}$.

\section{2-Benzyl-6-methoxybenzoic acid (9j)}

According to the general procedure, the recrystallized 2-methoxybenzoic acid $(11,0.76 \mathrm{~g}, 5 \mathrm{mmol})$ in THF was added dropwise to a stirred solution of $s$ BuLi/TMEDA (11 mmol). The solution was quenched by benzyl bromide (3.40 g, $20 \mathrm{mmol}$ ) (reverse addition). Workup in the usual manner followed by chromatography (cyclohexane/ethylacetate 80:20) afforded $9 \mathrm{j}$ (0.57 g, 47\%) as a white solid: mp $148-149.5^{\circ} \mathrm{C} .{ }^{1} \mathrm{H}$ NMR (200 MHz, $\left.\mathrm{CDCl}_{3}\right) \delta \mathrm{ppm}: 3.90(\mathrm{~s}, 3 \mathrm{H}), 4.14$ (s, 2H), $6.82(\mathrm{dd}, 2 \mathrm{H}, J=7.9 \mathrm{~Hz}, J=9.9 \mathrm{~Hz}), 7.16-7.34(\mathrm{~m}, 6 \mathrm{H}) .{ }^{13} \mathrm{C}$ NMR $(100 \mathrm{MHz}$, $\left.\mathrm{CDCl}_{3}\right) \delta$ ppm: 39.2, 56.1, 108.9, 109.2, 122.8, 126.3, 128.4 (2C), $129.2(2 \mathrm{C}), 131.2$, 140.0, 140.8, 156.8, 172.6. IR (neat): 2941, 1690, 1597, 1469, 1293, 1080, $932 \mathrm{~cm}^{-1}$.

\section{General procedure for the preparation of 3-substituted 2-methoxybenzoic acids (10a-f,h)}

2-Methoxybenzoic acid $(0.46 \mathrm{~g}, 3 \mathrm{mmol})$ in THF $(5 \mathrm{~mL})$ was added dropwise to a stirred solution of $n$-BuLi/t-BuOK (1:1 ratio, $12 \mathrm{mmol})$ in THF $(30 \mathrm{~mL})$ at $-78^{\circ} \mathrm{C}$. After being stirred $-78{ }^{\circ} \mathrm{C}$ for $2 \mathrm{~h}$, the reaction mixture was quenched with the electrophile (18 mmol) in THF (5 mL). Stirring was maintained for $1 \mathrm{~h}$ and the resulting mixture was allowed to warm to $\mathrm{rt}$ and hydrolyzed with water $(30 \mathrm{~mL})$. The aqueous layer was washed with diethyl ether $(2 \times 30 \mathrm{~mL})$, acidified with aqueous (2M) $\mathrm{HCl}$ until $\mathrm{pH}$ reached 1 , and extracted with diethyl ether $(3 \times 30 \mathrm{~mL})$. The organic layer was dried over $\mathrm{MgSO}_{4}$, filtrated and concentrated in vacuo to give the

10 Yang, K. L.; Balckman, B.; Diedrich, W.; Flaherty, P. T.; Mossman, C. J.; Roy, S.; Ahn, Y. M.;

Georg. G. I. J. Org. Chem. 2003, 68, 10030. 
crude benzoic acids (10a-f,h) which were purified by chromatography or fractional crystallization.

\section{2-Methoxy-3-methylbenzoic acid (10a)}

According to the general procedure, the recrystallized 2-methoxybenzoic acid (11, $0.46 \mathrm{~g}, 3 \mathrm{mmol})$ in THF was added dropwise to a stirred solution of $n-\mathrm{BuLi} / t-$ BuOK (1:1 ratio, $12 \mathrm{mmol})$. The solution was quenched by Mel $(2.55 \mathrm{~g}, 18 \mathrm{mmol})$. Workup in the usual manner followed by recrystallization (cyclohexane/ethylacetate) afforded 10a (0.199 g, 40\%) as a white solid: $\mathrm{mp} 81-83^{\circ} \mathrm{C} .{ }^{1} \mathrm{H}$ NMR $(200 \mathrm{MHz}$, $\mathrm{CDCl}_{3}$ ) $\delta$ ppm: 2.37 (s, 3H), 3.93 (s, 3H), 7.17 (dd, $1 \mathrm{H} \mathrm{J=7.8} \mathrm{Hz,} \mathrm{J=7.4} \mathrm{Hz),} 7.43$ (d, $1 \mathrm{H}, J=7.4 \mathrm{~Hz}), 7.91(\mathrm{~d}, 1 \mathrm{H}, J=7.8 \mathrm{~Hz}) .{ }^{13} \mathrm{C}$ NMR $\left(100 \mathrm{MHz}, \mathrm{CDCl}_{3}\right) \delta \mathrm{ppm:} \mathrm{16.0,}$ $62.1,122.2,124.8,130.6,131.9,136.9,158.1,167.6$. IR (neat): $2833,2575,1667$, 1589, 1468, 1307, 1089, $949 \mathrm{~cm}^{-1}$. HRMS (El) $\mathrm{m} / z$ calcd. for $\mathrm{C}_{9} \mathrm{H}_{10} \mathrm{O}_{3}\left(\mathrm{M}^{+}\right)$: 166.0629 . Found: 166.0628.

\section{2-Methoxy-3-(trimethylsilyl)benzoic acid (10b)}

According to the general procedure, the recrystallized 2-methoxybenzoic acid $(11,0.46 \mathrm{~g}, 3 \mathrm{mmol})$ in THF was added dropwise to a stirred solution of $n$-BuLi/tBuOK (1:1 ratio, $12 \mathrm{mmol})$. The solution was quenched by chlorotrimethylsilane (1.96 g, $18 \mathrm{mmol})$. Workup in the usual manner followed by chromatography (cyclohexane/ethylacetate 90:10) afforded 10b (0.269 g, 40\%) as a white solid: $\mathrm{mp}$ 96-98 ${ }^{\circ} \mathrm{C} .{ }^{1} \mathrm{H}$ NMR (400 MHz, $\left.\mathrm{CDCl}_{3}\right) \delta \mathrm{ppm}: 0.34(\mathrm{~s}, 9 \mathrm{H}), 3.89(\mathrm{~s}, 3 \mathrm{H}), 7.21(\mathrm{t}, 1 \mathrm{H}, J$ $=7.3 \mathrm{~Hz}), 7.64(\mathrm{dd}, 1 \mathrm{H}, J=7.3 \mathrm{~Hz}, J=1.8 \mathrm{~Hz}), 8.04(\mathrm{dd}, J=7.6 \mathrm{~Hz}, J=1.8 \mathrm{~Hz})$. ${ }^{13} \mathrm{C}$ NMR $\left(50 \mathrm{MHz}, \mathrm{CDCl}_{3}\right) \delta \mathrm{ppm}: 0.6(3 \mathrm{C}), 63.7,121.6,124.2,134.5,135.0,141.2$, 166.1, 170.6. IR (neat): 2947, 2651, 1677, 1579, 1452, 1298, 1127, $920 \mathrm{~cm}^{-1}$. HRMS (El) $m / z$ calcd. for $\mathrm{C}_{10} \mathrm{H}_{13} \mathrm{O}_{3} \mathrm{Si}\left(\mathrm{M}^{+}\right)$: 224.0868. Found: 224.0860. 


\section{3-Chloro-2-methoxybenzoic acid (10c)}

According to the general procedure, the recrystallized 2-methoxybenzoic acid $(11,0.46 \mathrm{~g}, 3 \mathrm{mmol})$ in THF was added dropwise to a stirred solution of $n-\mathrm{BuLi} / t$ BuOK (1:1 ratio, $12 \mathrm{mmol})$. The solution was quenched by hexachloroethane $(4.26 \mathrm{~g}$, $18 \mathrm{mmol})$. Workup in the usual manner followed by chromatography (cyclohexane/ethylacetate 80:20) afforded 10c (0.218 g, 39\%) as a yellow solid: $\mathrm{mp}$ 114-116 ${ }^{\circ} \mathrm{C} .{ }^{1} \mathrm{H}$ NMR (200 MHz, $\left.\mathrm{CDCl}_{3}\right) \delta \mathrm{ppm}: 4.06(\mathrm{~s}, 3 \mathrm{H}), 7.21(\mathrm{t}, 1 \mathrm{H}, J=8.0 \mathrm{~Hz})$, $7.62(\mathrm{dd}, 1 \mathrm{H}, J=8.0 \mathrm{~Hz}, J=1.6 \mathrm{~Hz}), 7.99(\mathrm{dd}, 1 \mathrm{H}, J=8.0 \mathrm{~Hz}, J=1.6 \mathrm{~Hz}) .{ }^{13} \mathrm{C} \mathrm{NMR}$ $\left(100 \mathrm{MHz}, \mathrm{CDCl}_{3}\right) \delta \mathrm{ppm}: 62.5,124.6,125.3,129.0,131.2,135.7,155.9,167.6 . \mathrm{IR}$ (neat): 2826, 2558, 1667, 1586, 1463, 1234, 1076, $917 \mathrm{~cm}^{-1}$. HRMS (EI) $m / z$ calcd. for $\mathrm{C}_{8} \mathrm{H}_{7} \mathrm{O}_{3} \mathrm{Cl}\left(\mathrm{M}^{+}\right)$: 186.0083. Found: 186.0079 .

\section{3-Bromo-2-methoxybenzoic acid (10d)}

According to the general procedure, the recrystallized 2-methoxybenzoic acid $(11,0.46 \mathrm{~g}, 3 \mathrm{mmol})$ in THF was added dropwise to a stirred solution of $n$-BuLi/tBuOK (1:1 ratio, $12 \mathrm{mmol})$. The solution was quenched by dibromotetrachloroethane (5.86 $\mathrm{g}, 18 \mathrm{mmol}$ ) (reverse addition). Workup in the usual manner followed by chromatography (cyclohexane/ethylacetate 80:20) afforded $10 \mathrm{~d}(0.235 \mathrm{~g}, 34 \%)$ as a white solid: $\mathrm{mp} 119-121^{\circ} \mathrm{C}$ (Lit. $\left.{ }^{(11)} 121^{\circ} \mathrm{C}\right) .{ }^{1} \mathrm{H}$ NMR $\left(200 \mathrm{MHz}, \mathrm{CDCl}_{3}\right) \delta$ ppm: 4.07 (s, 3H), 7.22 (t, $1 \mathrm{H}, J=8.0 \mathrm{~Hz}), 7.63$ (dd, $1 \mathrm{H}, J=8.0 \mathrm{~Hz}, J=1.5 \mathrm{~Hz}), 8.01$ (dd, $1 \mathrm{H}$, $J=8.0 \mathrm{~Hz}, J=1.5 \mathrm{~Hz}) \cdot{ }^{13} \mathrm{C}$ NMR $\left(100 \mathrm{MHz} \mathrm{CDCl}_{3}\right) \delta \mathrm{ppm}: 61.6,124.9,125.1,128.2$, 129.5, 133.4, 154.5, 166.4. IR (neat): 2952, 1669, 1588, 1465, 1222, 1077, $992 \mathrm{~cm}^{-1}$.

\section{3-lodo-2-methoxybenzoic acid (10e)}

According to the general procedure, the recrystallized 2-methoxybenzoic acid $(11,0.46 \mathrm{~g}, 3 \mathrm{mmol})$ in THF was added dropwise to a stirred solution of $n$-BuLi/tBuOK (1:1 ratio, $12 \mathrm{mmol})$. The solution was quenched by iodine $(4.57 \mathrm{~g}, 18 \mathrm{mmol})$ (reverse addition). Workup in the usual manner followed by chromatography

\footnotetext{
11 Pudleiner, H.; Laatsch, H. Synthesis 1989, 4, 286.
} 
(cyclohexane/ethylacetate 80:20) afforded 10e (0.225 g, 27\%) as a white solid: $\mathrm{mp}$ 122-124 ${ }^{\circ} \mathrm{C} .{ }^{1} \mathrm{H}$ NMR (200 MHz, $\left.\mathrm{CDCl}_{3}\right) \delta$ ppm: $3.99(\mathrm{~s}, 3 \mathrm{H}), 7.01(\mathrm{t}, 1 \mathrm{H}, J=7.8 \mathrm{~Hz})$, 8.00-8.07 (m, 2H). ${ }^{13} \mathrm{C}$ NMR (100 MHz, $\left.\mathrm{CDCl}_{3}\right) \delta$ ppm: 62.8, 93.1, 123.9, 126.4, 132.3, 144.9, 159.6, 168.1. IR (neat): 2924, 1673, 1581, 1456, 1402, 1296, 1074, 992 $\mathrm{cm}^{-1}$.

\section{2-Methoxy-3-(methylthio)benzoic acid (10f)}

According to the general procedure, the recrystallized 2-methoxybenzoic acid $(11,0.46 \mathrm{~g}, 3 \mathrm{mmol})$ in THF was added dropwise to a stirred solution of $n$-BuLi/tBuOK (1:1 ratio, $12 \mathrm{mmol})$. The solution was quenched by dimethyl disulfide $(1.69 \mathrm{~g}$, $18 \mathrm{mmol}$ ). Workup in the usual manner followed by recrystallization (cyclohexane/ethylacetate) afforded $10 \mathrm{f}(0.27 \mathrm{~g}, 45 \%)$ as a white solid: $\mathrm{mp} 77-79{ }^{\circ} \mathrm{C}$. ${ }^{1} \mathrm{H}$ NMR (200 MHz, $\mathrm{CDCl}_{3}$ ) $\delta$ ppm: 2.49 (s, 3H), 4.03 (s, 3H), 7.25 (t, 1H, J = 7.8 Hz), 7.42 (dd, $1 \mathrm{H}, J=7.8 \mathrm{~Hz}, J=1.6 \mathrm{~Hz}$ ), 7.89 (dd, $J=7.8 \mathrm{~Hz}, J=1.6 \mathrm{~Hz}) .{ }^{13} \mathrm{C} \mathrm{NMR}$ $\left(100 \mathrm{MHz}, \mathrm{CDCl}_{3}\right) \delta$ ppm: 14.8, 61.7, 124.5, 124.9, 128.3, 130.1, 134.2, 156.1, 168.5. HRMS (EI) $m / z$ calcd. for $\mathrm{C}_{9} \mathrm{H}_{10} \mathrm{O}_{3} \mathrm{~S}\left(\mathrm{M}^{+}\right)$: 198.0350. Found: 198.0339 .

\section{3-(Hydroxy(phenyl)methyl)-2-methoxybenzoic acid (10h)}

According to the general procedure, the recrystallized 2-methoxybenzoic acid $(11,0.46 \mathrm{~g}, 3 \mathrm{mmol})$ in THF was added dropwise to a stirred solution of $n-\mathrm{BuLi} / t$ BuOK (1:1 ratio, $12 \mathrm{mmol})$. The solution was quenched by benzaldehyde $(1.91 \mathrm{~g}$, $18 \mathrm{mmol}$ ) (reverse addition). Workup in the usual manner followed by chromatography (cyclohexane/ethylacetate 70:30) afforded 10h $(0.318 \mathrm{~g}, 41 \%)$ as a white solid: $\mathrm{mp} 124-126{ }^{\circ} \mathrm{C} .{ }^{1} \mathrm{H}$ NMR $\left(200 \mathrm{MHz}, \mathrm{CDCl}_{3}\right) \delta \mathrm{ppm}: 3.72$ (s, 3H), 6.16 (s, 1H), 7.23-7.30 (m, 3H), 7.31-7.38 (m, 3H), 7.69 (dd, 1H, J=7.6 Hz, J=1.5 Hz), 7.98

(dd, $1 \mathrm{H}, J=7.8 \mathrm{~Hz}, J=1.5 \mathrm{~Hz}) \cdot{ }^{13} \mathrm{C}$ NMR $\left(100 \mathrm{MHz}, \mathrm{CDCl}_{3}\right) \delta \mathrm{ppm}: 63.1,71.0,124.4$, 126.6, 127.7, 128.5, 132.2, 133.4, 138.3, 143.1, 158.0, 171.5. IR (neat): 3427, 2939, $1708,1590,1429,1210,992,752 \mathrm{~cm}^{-1}$. 


\section{C- Synthesis of Iunularic acid (18)}

\section{2-Methoxy-6-(4-methoxyphenethyl)benzoic acid}

To a solution of LDA $(6 \mathrm{mmol})$ in dry THF $(10 \mathrm{~mL})$ at $-78^{\circ} \mathrm{C}$ was added dropwise 2-methoxy-6-methylbenzoic acid (9a) (0.25 g, $1.5 \mathrm{mmol})$ in THF (4 mL). After $2 \mathrm{~h}$ at this temperature, the mixture was quenched by 1-(chloromethyl)-4methoxybenzene $(1.22 \mathrm{~mL}, 9 \mathrm{mmol})$. The resulting solution was stirred for 2 additional hours, and was then slowly allowed to warm to ambient temperature, after which water $(30 \mathrm{~mL})$ was added. The aqueous layer was washed with diethyl ether $(2 \times 30 \mathrm{~mL})$, and shaken, and then acidified with $2 \mathrm{M} \mathrm{HCl}$. The mixture was diluted with diethyl ether and the organic layer was separated and dried with $\mathrm{MgSO}_{4}$. Filtration and concentration in vacuo gave crude 2-methoxy-6-(4methoxyphenethyl)benzoic acid as a viscous oil which was used as such for the next step. The yield was estimated to be $>99 \%$ by ${ }^{1} \mathrm{H}$ NMR. ${ }^{1} \mathrm{H}$ NMR $\left(200 \mathrm{MHz}, \mathrm{CDCl}_{3}\right) \delta$ ppm: 2.78-2.91 (m, 2H), 2.97-3.06 (m, 2H), 3.76 (s, 3H), 3.89 (s, 3H), 6.77-6.85 (m, $4 \mathrm{H}), 7.09-7.10(\mathrm{~m}, 2 \mathrm{H}), 7.22(\mathrm{~m}, 1 \mathrm{H}) .{ }^{13} \mathrm{C} \mathrm{NMR}\left(50 \mathrm{MHz}, \mathrm{CDCl}_{3}\right) \delta \mathrm{ppm:} \mathrm{36.5,} \mathrm{36.9,}$ 55.3, 56.1, 109.1, 113.9 (2C), 122.3, 129.5 (2C), 131.1, 133.8, 141.2, 156.7, 157.9, 173.4. IR (neat): $3004,2932,1714,1662,1584,1512,1469,1232,1075,795 \mathrm{~cm}^{-1}$.

\section{2-Hydroxy-6-(4-hydroxyphenethyl)benzoic acid (lunularic acid (18))}

Boron tribromide (13.5 mL, $13.5 \mathrm{mmol}, 1 \mathrm{M}$ in dichloromethane) was added dropwise to a stirred solution of crude 2-methoxy-6-(4-methoxyphenethyl)benzoic acid $(0.43 \mathrm{~g}, 1.5 \mathrm{mmol})$ in dichloromethane $(15 \mathrm{~mL})$ under argon at $-78{ }^{\circ} \mathrm{C}$. After being stirred for $1 \mathrm{~h}$ at this temperature, the solution was allowed to warm up to rt overnight. Water $(10 \mathrm{~mL})$ was added and the aqueous layer was extracted with dichloromethane $(2 \times 10 \mathrm{~mL})$. The solvent was removed in vacuo and the residue was chromatographed (ethyle acetate/cyclohexane/acetic acid 30:65:5) to afford 2hydroxy-6-(4-hydroxyphenethyl)benzoic acid (lunularic acid (18)) as a white solid 
(0.34 g, 87\%): mp 195-197 ${ }^{\circ} \mathrm{C}\left(\right.$ Lit. $\left.^{(12)} 199-200{ }^{\circ} \mathrm{C}\right) .{ }^{1} \mathrm{H}$ NMR $\left(200 \mathrm{MHz}, \mathrm{CDCl}_{3}\right) \delta$ ppm: 2.79-3.00 (m, 2H), 3.21-3.37 (m, 2H), 6.62-6.77 (m, 4H), $6.98(\mathrm{t}, 2 \mathrm{H}, J=8 \mathrm{~Hz}), 7.16$ $(\mathrm{t}, 1 \mathrm{H}, J=8.0 \mathrm{~Hz}) \cdot{ }^{13} \mathrm{C} \mathrm{NMR}\left(100 \mathrm{MHz}\right.$, acetone- $\left.d_{6}\right) \delta \mathrm{ppm}: 37.9,39.2,112.6,115.4$ (2C), 115.8, 122.8, 129.7 (2C), 133.3, 134.4, 145.9, 155.8, 163.2, 173.2. IR (neat): $2350-3360,1685,1581,1471,1266,1061,918,745 \mathrm{~cm}^{-1}$. 